\title{
Implementation of optical flow: good feature definition for tracking of heart cavity
}

\author{
Anwar ${ }^{1}$, Riyanto Sigit ${ }^{2}$, Achmad Basuki ${ }^{3}$, I Putu Adi Surya Gunawan ${ }^{4}$ \\ ${ }^{1,2,3}$ Department of Informatics Engineering, Politeknik Elektronika Negeri Surabaya, Indonesia \\ ${ }^{4}$ Department of Electrical Engineering, Politeknik Elektronika Negeri Surabaya, Indonesia
}

\begin{abstract}
Article Info
ABSTRACT

Article history:

Received Sep 10, 2019

Revised Nov 11, 2019

Accepted Nov 25, 2019

\section{Keywords:}

Echocardiography

Good feature

Image enhancement

Image segmentation

Optical flow

Echocardiography is a method of examination using high-frequency sound waves to capture images of the heart organ structure. Echocardiography video is used by a doctor to analyze heart wall cavity movements and identify heart disease. Several points of view including the long axis, the two and four cavities in the left ventricle can be used in the examination of heart function. Cardiac assessment is still performed conventionally, which requires a level of thoroughness. This research proposes a method for tracking the movement of the heart wall. In this method, the good feature was defined only in the first frame. Furthermore, the whole frame will be processed by the optical flow method. Good feature definition consists of image enhancement, segmentation and tracking processes using the optical flow. Furthermore, the calculation of contour similarity from the proposed method by forming contour manually using 24 point initialization to draw the heart cavity. The proposed method shows the calculation results with a sensitivity of $90 \%$ and an accuracy of $87.451 \%$.
\end{abstract}

Copyright $(2020$ Institute of Advanced Engineering and Science. All rights reserved.

\section{Corresponding Author:}

Anwar,

Department of Informatics Engineering,

Politeknik Elektronika Negeri Surabaya, Indonesia.

Email: anwar@pasca.student.pens.ac.id

\section{INTRODUCTION}

The heart is a vital part of the human body that receives and pumps blood throughout the body. The main function of the heart is pumping blood to the vessels to channel oxygen and nutritional components. According to the American Heart Association (AHA), several points of view, including the long axis, two and four cavities in the left ventricle are recommended to observe in determining the heart abnormalities. Observations were made to assess the movement of the heart when contracting, relaxing, and thickening each segment. The assessment resulted in 3 criteria, namely normal, hypokinetic and akinetic [1].

Echocardiography is an examination method using high-frequency sound waves to capture images of the structure of the heart organ. Examination of heart conditions using echocardiography as an imaging method is used to detect the potential for heart disease, thus that appropriate treatment can be decided and also used to evaluate treatment. Examination of heart conditions typically using Simpson's method to determine the volume of the left ventricle during systole to the diastole phase. The Simpson method is only used in parts two and four of the left ventricular chamber. The Simpson method is the most commonly used approach to measure the left ventricular function. This method involves a manual search of the end-systole and end-diastole, from the apical four-chamber and apical two-chamber [2, 3].

However, in clinical practice, you will face situations where the Simpson method still uses conventional methods and requires a level of thoroughness. The main limitation this method is poor image quality, which allows the unreliable formation of endocardial contours. The echocardiographic image needs to be improved since it contains noise and has unbalanced brightness that can interfere with the examination. 
This type of noise in echocardiographic images is called speckle noise, which is caused by an error in the process of transmitting data, causing image data to produce noise. The application of various ultrasonographic image processing techniques has been explored in the literature, most of those techniques purposed to reduce speckle noise by maintaining image structure.

The evaluation uses a segmentation process that forms the contour of the left ventricular heart wall cavity, has been implemented using several methods. Nakphu [4] segmented the apical four-chamber view using the watershed method and compared the results of segmentation with active contour-based. The results show that the watershed transform performs better than active contour. Sigit [5] segmented the heart cavity automatically on the short axis view using the active shape model method. A study of conducted segmentation automatically using the triangle method, this method segmentation obtained the smallest value of the error evaluation of $8.18 \%$, the snake method of $19.94 \%$ and the watershed of $15.97 \%$ [6, 7]. Anwar [8] proposed the development of a high-boost filter method to improve the results of heart segmentation in two and four chambers, and obtain an accuracy of $89.409 \%$.

An assessment of the condition of the heart can be seen using the optical flow method to track the movement of diastole to the systole, thickening of the heart wall indicate cardiac abnormalities [9]. A study of using optical flow as a tracking system on the movement of the heart wall. Sigit $[10,11]$ semi-automatic segmentation using initialization point as a good feature for tracking the movement of the heart using optical flow. This method is also used in the apical four-chamber view heart movement tracking system which can reconstruct the heart cavity wall lines with a semi-automatic system [12]. Aziz [13] proposed the development on echocardiography images to segmentation take the boundary of endocardium of left ventricular in short axis cardiac, however still semi-automatic for approach on detecting the contour of endocardium as good features in Lucas-Kanade optical flow. Sengar [14] proposes a new and efficient approach to moving detection objects under a static background, using the proposed block-based three-frame differencing method almost all background noises are removed thus target detection result is optimal.

Based on previous research that discusses the tracking system in the part of the heart cavity semiautomatically, most of those research have not yet been processed parts of the left ventricle automatically at the view of two-chamber and four-chambers. This research uses an image processing approach $[8,13,14]$ to find good features in echocardiographic videos automatically. Good feature definition consists of image enhancement, segmentation, intersection and tracking processes using the optical flow. In this method, a good feature was defined only in the first frame. Furthermore, the whole frame will be processed by the optical flow method. Good features will be continued using optical flow to detect and track the movement of the heart wall. This study proposes the development of a heart wall cavity tracking system automatically using optical flow.

\section{RESEARCH METHOD}

This study focuses on defining good features automatically. The good features are used as initialization point in optical flow to track the movement of the heart wall. In this section, we describe the procedure for definition of good features. This method consists of image enhancement, segmentation and tracking processes using optical flow. Figure 1 shows the system diagram that has been developed to implement the objectives of this study.

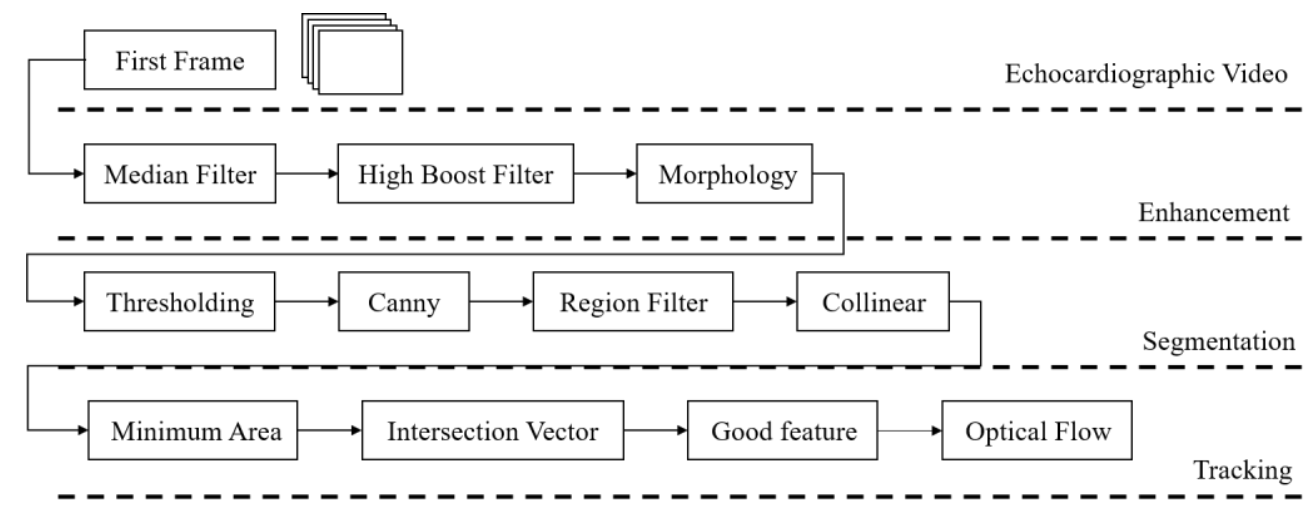

Figure 1. System design 


\subsection{Heart Movement Assessment}

Assessment of function in the left ventricle is usually by estimating the percentage of blood released during the final systole process with the total final diastole volume. Heart condition assessment can also be done visually based on the left ventricular movement. Pandian [9] describes the asymmetrical movement of the left ventricular wall, giving an indication of cardiac abnormalities. According to Catherine Otto [15], there are four qualitative scales used for assessment in the movement of the heart wall. Qualitative scale of assessment of heart movement as shown in Table 1.

Table 1. Qualitative Scale of Assessment of Heart Movement

\begin{tabular}{ccc}
\hline No & $\begin{array}{c}\text { Wall } \\
\text { Movement }\end{array}$ & Defintion \\
\hline 1 & $\begin{array}{c}\text { Normal } \\
\text { Hypokinetics }\end{array}$ & $\begin{array}{c}\text { Movement of the heart wall towards the center and thickening of the wall during systole } \\
\text { The amplitude of the heart wall movement during systole decreases }<5 \mathrm{~mm}, \text { delays in the onset of } \\
\text { contractions and relaxation }\end{array}$ \\
3 & $\begin{array}{c}\text { Akinetic } \\
\text { Dyskenetic }\end{array}$ & Movement of the heart wall to the center during systole does not occur \\
Movement out during systole
\end{tabular}

\subsection{Image Enhancement}

The difference between the wall and the heart cavity produced by an echocardiographic device is not too clear for an identification. Therefore, the difference between the wall and the heart cavity needs to be strengthened. The echocardiographic image needs to be improved since it contains speckle noise and has unbalanced brightness that can interfere with the examination. The use of a median filter deemed suitable for this case. The working principle of the median filter is spatial processing to determine the pixels in the image that are affected by speckle noise[8]. After the speckle noise was reduced, the clarity of the image still needs to improve, to clarify the part between the wall and the heart cavity. High-boost filters are used to improve high-frequency components while maintaining low-frequency components. This High-boost filter sharpens the edges of images and obtains clearer images [16]. The high-boost filter can be expressed as $G_{(m, n)}$, where $A$ is the gain and $F_{(m, n)}$ is the original image.

$$
\begin{aligned}
& G_{(m, n)}=A \cdot F_{(m, n)}-\operatorname{lowpass}\left(F_{(m, n)}\right) \\
& G_{(m, n)}=(A-1) \cdot F_{(m, n)}+\left[F_{(m, n)}-\operatorname{lowpass}\left(F_{(m, n)}\right)\right] \\
& G_{(m, n)}=(A-1) \cdot F_{(m, n)}+\operatorname{highpass}\left(F_{(m, n)}\right)
\end{aligned}
$$

A morphology operation was performed in order to reduce the noise in a grayscale image from the result of a high-boost filter. Morphology is used to improve the shape of images, a combination of morphology opening (4) and closing (5) was used to deal with noise. Morphology opening and closing can be expressed as $A \circ B, A \bullet B$, where $A$ is the result high-boost filter and $B$ is the structuring element.

$$
\begin{aligned}
& A \circ B=(A \oplus(-B)) \oplus B \\
& A \bullet B=(A \oplus B) \oplus(-B)
\end{aligned}
$$

\subsection{Image Segmentation}

Approach to image processing with a background subtraction can detect moving targets efficiently and effectively as compared to the traditional approaches [17]. This study uses an image segmentation approach to obtain cavity contours as a good feature. In this method, the good feature was defined only in the first frame. Furthermore, the whole frame will be processed by optical flow method. Image segmentation stage uses thresholding, canny, region area and collinear methods to get the contour of the heart cavity [8]. Global thresholding method was used to perform segmentation of the heart cavity. The result of global thresholding is a binary image which has a difference as a boundary between two different regions in images. Global thresholding can be expressed as $g(x, y)$, where $p(x, y)$ is the input image and $T$ is the threshold. 


$$
g(x, y)=\left\{\begin{array}{l}
0, p(x, y)<T \\
1, p(x, y) \geq T
\end{array}\right.
$$

The Canny filter is an algorithm that used to detect edges on shape objects contained in an image. Canny filters can be expressed by (7-10).

$$
\begin{aligned}
& \text { Smooth by Gaussian } \quad S=G \sigma^{*} I \quad G \sigma=\frac{1}{\sqrt{2 \pi \sigma}} \exp \left(-\frac{x^{2}+y^{2}}{2 \sigma^{2}}\right) \\
& \text { Compute } x \text { and y derivatives } \quad \nabla S=\left[\frac{\partial}{\partial x} S \frac{\partial}{\partial y} S\right]=\left[S_{x} S_{Y}\right]^{T} \\
& \text { Compute gradient magnitude } \\
& \text { and orientation } \\
& |\nabla S|=\sqrt{S_{x}^{2}-S_{y}^{2}} \quad \theta=\tan ^{-1} \frac{S_{y}}{S_{x}}
\end{aligned}
$$

Threshold

$$
|\nabla S| \geq \text { Threshold }
$$

The results of detection using a canny filter have not been able to obtain the desired contour, there is a small noise in some parts. To remove the noise, a region area calculation was performed to eliminate the area with low value. Contour which has an area of less than 100, will be removed considering it is not part of the heart wall cavity. Region area as shown in Figure 2.

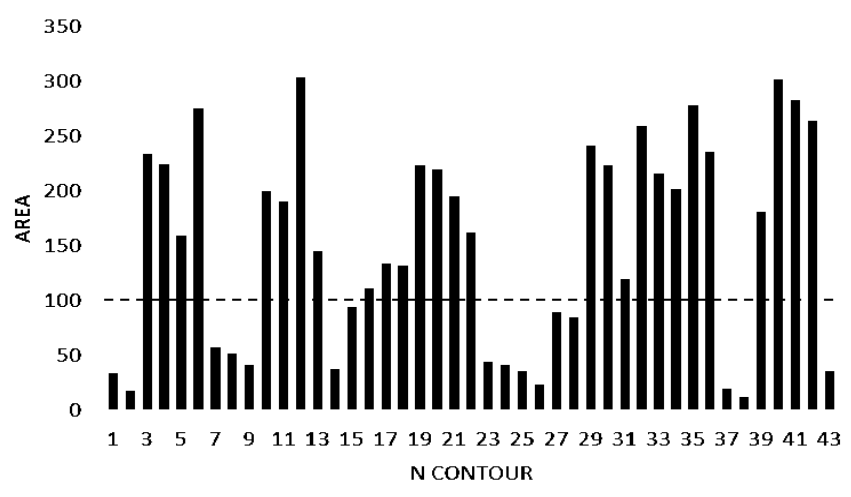

Figure 2. Region area

The results from the region area indicate that a large part of the noise cannot be removed, thus an improvement needs to be done to remove large noise in the contour area using the collinear method. The equation of collinear described in (11). The collinear method is applied if there are three or more points in a straight line [13]. The third one will be detected as an unnecessary point and will be removed.

$$
x_{1}\left(y_{2}-y_{3}\right)+x_{2}\left(y_{3}-y_{1}\right)+x_{3}\left(y_{1}-y_{2}\right)=0
$$

\subsection{Good Feature Definition}

The heart cavity contour that has been obtained from the segmentation method will be good features in optical flow. However not all contour lines are used for tracking in the next frame, the method proposed in this study is to find the distribution of contour points. The points obtained are good feature is used as an initialization point in optical flow to track the movement of the heart wall. Approach to obtain good feature stage uses minimum area and intersection vector. The method of minimum area is an algorithm that can be 
used to solve optimization problems including finding the width or diameter of a contour. This function calculates the angle of each point between the side of the square located above and the edge of the contour [18]. The working principle of minimum-area with rotating rectangle squares at each corner to find the minimum value and calculate area $A_{1}$, update the minimum area and save the current rectangle if $A_{1}<A_{\min }$.

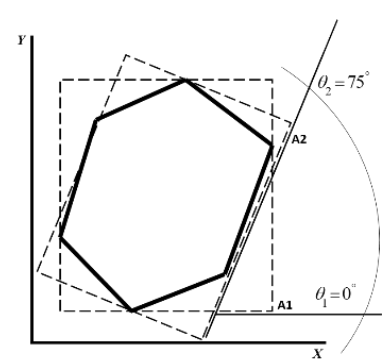

(a)

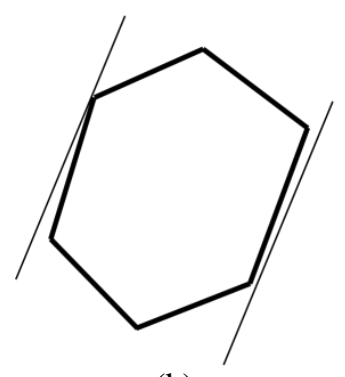

(b)

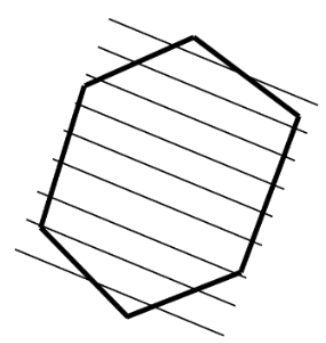

(c)

Figure 3. (a) Minimum area, (b) Minimum area result, (c) Intersection vector

Intersecting lines are used to define good features, which are obtained from the intersection of lines on the contour by taking a minimum line of rectangular on the left and right sides. The good features are used as initialization points in optical flow to track the movement of the heart wall.

\subsection{Optical Flow}

Movements in the space can be described as areas of motion and are embodied through the gradient of different grayscale distribution. The motion field in space is transformed into an image, is known as an optical flow field that shows techniques for characterizing image motion [19]. The working principle of optical flow is neighboring pixels have similar motion, and pixel intensities of an object do not change between consecutive frames. Optical flow works on several assumptions, the pixel intensities of an object do not change between consecutive frames and neighboring pixels have similar motion. Where, consider a pixel $I(x, y, t)$ in first frame and it moves by distance $(\Delta x, \Delta y)$ in next frame taken after $\Delta t$, thus since those pixels are the same and intensity does not change, can be expressed by (12).

$$
I(x, y, t)=I(x+\Delta x, y+\Delta y, t+\Delta t)
$$

Then take taylor series approximation of right-hand side, remove common terms and divide by $\Delta t$ to get the following (13):

$$
\begin{array}{r}
f_{x}=\frac{\partial f}{\partial x} ; f_{y}=\frac{\partial f}{\partial y} \\
f_{x} u+f_{y} v+f_{t}=0 \text { Where } \begin{array}{l}
\Delta x \\
u=
\end{array} v=\frac{\Delta y}{\Delta t}
\end{array}
$$

As shown in (13) is called optical flow equation, Therein can be found $f_{x}$ and $f_{y}$ they are image gradients. Similarly, $f_{t}$ is the gradient along time. However, the $(u, v)$ value is unknown. Therefore several methods are provided to solve this problem and one of them is Lucas-Kanade. This method uses a $3 \times 3$ patch around the point. Thus all the 9 point have the same motion. Find $\left(f_{x}, f_{y}, f_{t}\right)$ for these 9 points. Below is the final solution which is two equation-two unknown problem and solve to get the solution.

$$
\left[\begin{array}{l}
u \\
v
\end{array}\right]=\left[\begin{array}{lr}
\sum_{i} f_{x_{i}}{ }^{2} & \sum_{i} f_{x_{i}}, f_{y_{i}} \\
\sum_{i} f_{x_{i}}, f_{y_{i}} & \sum_{i} f_{y_{i}}{ }^{2}
\end{array}\right]^{-1}\left[\begin{array}{l}
-\sum_{i} f_{x_{i}}, f_{t_{i}} \\
-\sum_{i} f_{y_{i}}, f_{t_{i}}
\end{array}\right]
$$




\section{RESULTS AND ANALYSIS}

\subsection{Image Enhancement Result}

The results of the image enhancement process in each step are shown in Figure 4. The echocardiographic image needs to be improved since it contains noise and has unbalanced brightness that can interfere with the examination. This type of noise in echocardiographic images is called speckle noise, which is caused by an error in the process of transmitting data, causing image data has a lot of noise. First is to improve the image using a median filter with $27 \times 27$ kernels to reduce speckle noise in echocardiography images. The use of large kernel results in blurred images, however still retaining the image edge portion and improve the line edge cavity [8]. High-boost filter and morphology operation are used to clarify the difference between the cavity and heart wall. In this method, the heart cavity will become darker and the heart wall will become brighter.

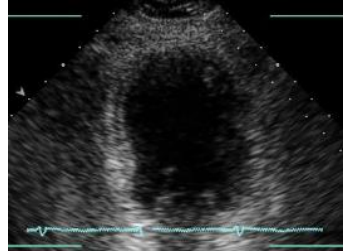

(a)

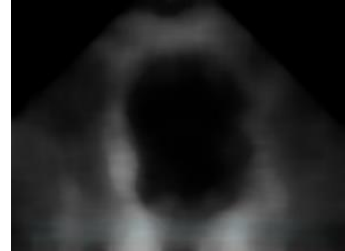

(b)

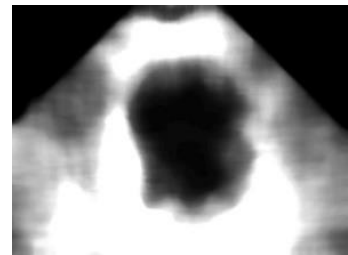

(c)

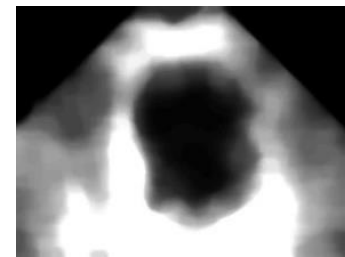

(d)

Figure 4. Image enhancement result. (a) original image, (b) median filter result,

(c) high-boost filter result, (d) morphology result

\subsection{Image Segmentation Result}

The results of the image segmentation process in each step are shown in Figure 5. Once the difference between the cavity and heart wall clearly visible, the next step is performing the segmentation process using global thresholding. Global thresholding was used to segment the heart cavity. This method produces a binary image that has a line as a boundary between two different regions in an image. The Canny filter is used to obtain an edge line in the heart cavity. The results of detection using a canny filter have not been able to obtain the desired contour, there is small contour in some point of noise. Region area and collinear are used to remove some contour that is not categorized as a heart cavity [20].

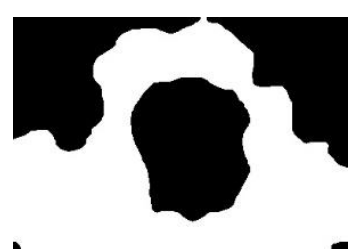

(a)

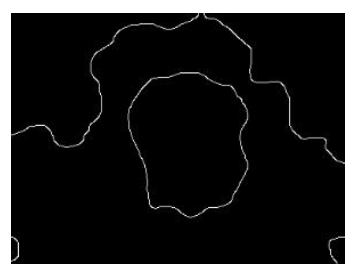

(b)

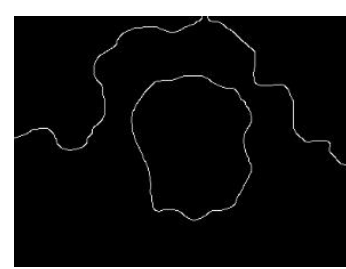

(c)

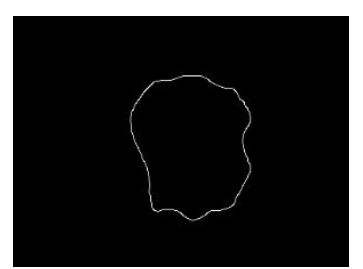

(d)

Figure 5. Image segmentation result. (a) global thresholding result, (b) canny filter result, (c) region area result, (d) collinear result

\subsection{Good Feature \& Optical Flow}

A study of finding good features to track [21] using optical flow show that the proposed feature quality measure highly correlates with the accuracy in structure and motion estimation. In this research, the good feature was defined only in the first frame. Approach to obtain good feature stage uses minimum area and intersection vector. The results of the find a good feature process in each step are shown in Figure 6. Intersecting lines are used to define good features, which are obtained from the intersection of lines on the contour by taking a minimum line of rectangular on the left and right sides. The good features are used as initialization point in optical flow to track the movement of the heart wall. 


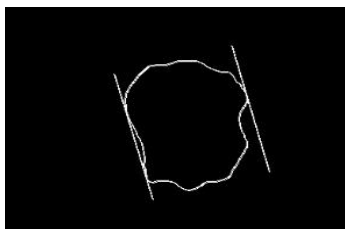

(a)

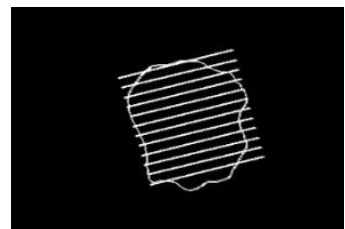

(b)

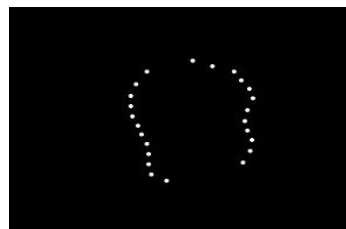

(c)

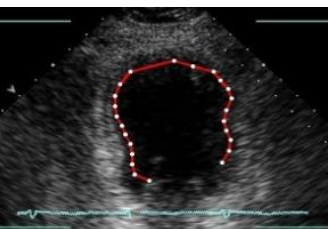

(d)

Figure 6. Good feature result. (a) minimum area result, (b) intersection vector result, (c) good feature result, (d) boundary result

This research proposed the development of a heart wall cavity tracking system automatically using optical flow. Finding good features in echocardiographic videos automatically from the formation of cavity contours. Good features will be continued using optical flow to detect and track the movement of the heart wall. Figure 7. shows the proposed method can track well the movement of the heart wall, which is marked with red contours.

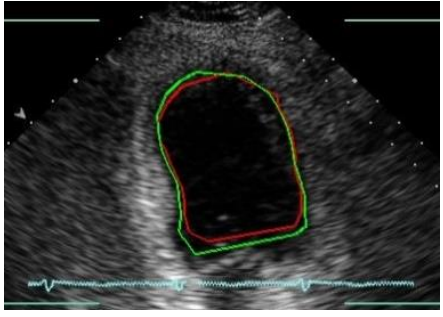

(a)

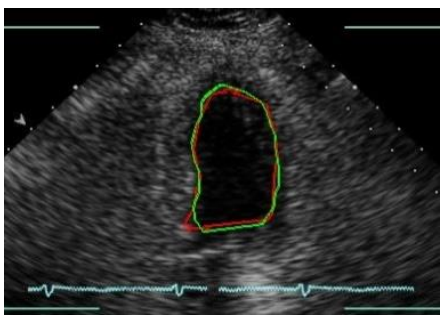

(d)

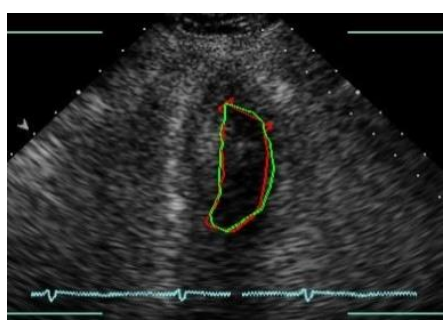

(g)

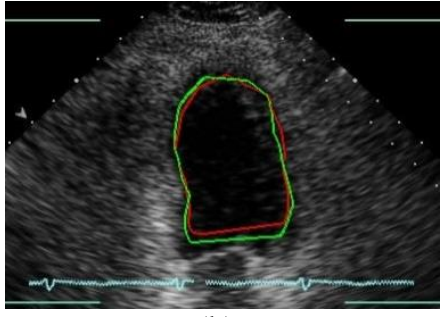

(b)

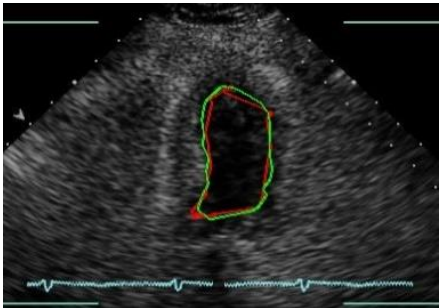

(e)

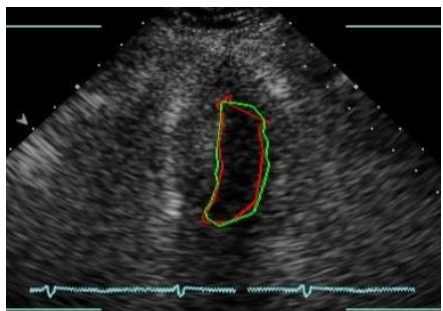

(h)

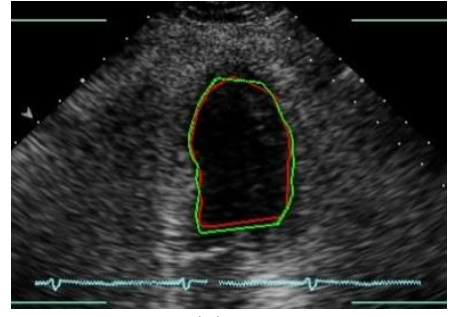

(c)

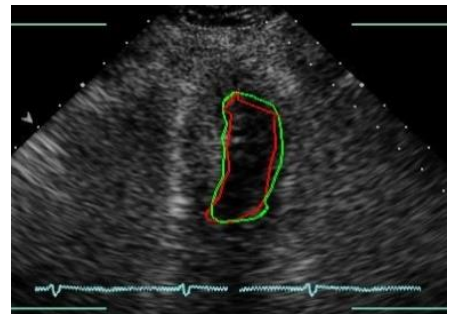

(f)

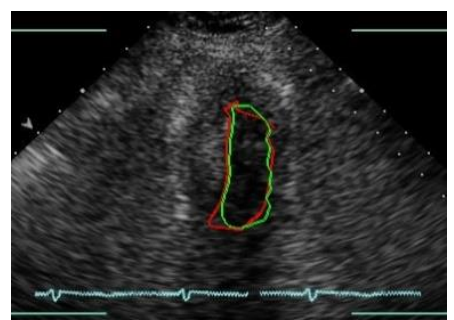

(i)

Figure 7. Tracking optical flow \& manual. (a) frame 1, (b) frame 2, (c) frame 3, (d) frame 4, (e) frame 5, (f) frame 6, (g) frame 7, (h) frame 8, (i) frame 9

After several experiments, a discussion about result of heart cavity image processing, definition good feature and tracking processes using the optical flow. Furthermore, the calculation of contour similarity from the proposed method by forming contour manually using 24 point initialization to draw the heart cavity. The measurement uses 6 echocardiographic video datasets, each of which has 10 frames. Figure 7 shows the comparison results of manually formed contours (green line) and contour formation from tracking results with the proposed method (red line). If there is a red contour line which is on the contour area green line then as true positive (TP). If there is a red contour line on outside the contour area green line then as false positive (FP). If there is a red contour line that is not in the contour area green line then as false negative (FN). 
Based on Table 2, 3, 4, 5, 6, 7 proposed method shows the calculation results with a sensitivity of $90 \%$ and an accuracy of $87.451 \%$.

Table 2. Measured Contour Area Data 1

\begin{tabular}{cccccc}
\hline Frame & \multicolumn{2}{c}{ Area Contour(Pixel) } & Sensitivity & Accuracy \\
Video & TP & FP & FN & $(\%)$ & $(\%)$ \\
\hline 1 & 15858 & 365 & 982 & 94.17 & 92.17 \\
2 & 14627 & 161 & 1901 & 88.50 & 87.64 \\
3 & 11341 & 247 & 1349 & 89.37 & 87.66 \\
4 & 9749 & 43 & 1013 & 90.59 & 90.23 \\
5 & 7793 & 238 & 632 & 92.50 & 89.96 \\
6 & 5471 & 82 & 804 & 87.19 & 86.06 \\
7 & 4185 & 78 & 1226 & 77.34 & 76.24 \\
8 & 3936 & 200 & 480 & 89.13 & 85.27 \\
9 & 3619 & 104 & 772 & 82.42 & 80.51 \\
10 & 3448 & 606 & 290 & 92.24 & 79.37 \\
\hline
\end{tabular}

Table 4. Measured Contour Area Data 3

\begin{tabular}{cccccc}
\hline $\begin{array}{c}\text { Frame } \\
\text { Video }\end{array}$ & \multicolumn{2}{c}{ Area Contour(Pixel) } & $\begin{array}{c}\text { Sensitivity } \\
(\%)\end{array}$ & $\begin{array}{c}\text { Accuracy } \\
(\%)\end{array}$ \\
\hline 1 & 12506 & 211 & 1290 & 90.65 & 89.28 \\
2 & 13236 & 74 & 1314 & 90.97 & 90.51 \\
3 & 13433 & 460 & 915 & 93.62 & 90.71 \\
4 & 12191 & 289 & 1169 & 91.25 & 89.32 \\
5 & 11073 & 439 & 875 & 92.68 & 89.39 \\
6 & 10124 & 230 & 808 & 92.61 & 90.70 \\
7 & 8969 & 178 & 1462 & 85.98 & 84.54 \\
8 & 7746 & 75 & 1533 & 83.48 & 82.81 \\
9 & 6913 & 166 & 1090 & 86.38 & 84.62 \\
10 & 6229 & 13 & 1585 & 79.72 & 79.58 \\
\hline
\end{tabular}

Table 6. Measured Contour Area Data 5

\begin{tabular}{cccccc}
\hline Frame & \multicolumn{2}{c}{ Area Contour(Pixel) } & Sensitivity & Accuracy \\
Video & TP & FP & FN & $(\%)$ & $(\%)$ \\
\hline 1 & 22908 & 3539 & 239 & 98.97 & 85.84 \\
2 & 22064 & 2788 & 898 & 96.09 & 85.69 \\
3 & 22608 & 1428 & 726 & 96.89 & 91.30 \\
4 & 20429 & 1680 & 693 & 96.72 & 89.59 \\
5 & 18981 & 2121 & 319 & 98.35 & 88.61 \\
6 & 17377 & 2158 & 553 & 96.92 & 86.50 \\
7 & 15286 & 2081 & 755 & 95.29 & 84.35 \\
8 & 14081 & 2256 & 479 & 96.71 & 83.74 \\
9 & 12635 & 1717 & 655 & 95.07 & 84.19 \\
10 & 12227 & 1456 & 1196 & 91.09 & 82.18 \\
\hline
\end{tabular}

Table 3. Measured Contour Area Data 2

\begin{tabular}{cccccc}
\hline Frame & \multicolumn{2}{c}{ Area Contour(Pixel) } & Sensitivity & Accuracy \\
Video & TP & FP & FN & $(\%)$ & $(\%)$ \\
\hline 1 & 18203 & 19 & 1831 & 90.86 & 90.77 \\
2 & 17718 & 185 & 1812 & 90.72 & 89.87 \\
3 & 17372 & 72 & 2031 & 89.53 & 89.20 \\
4 & 16849 & 49 & 2695 & 86.21 & 85.99 \\
5 & 15905 & 90 & 1993 & 88.86 & 88.42 \\
6 & 15456 & 46 & 2042 & 88.33 & 88.10 \\
7 & 14667 & 39 & 1981 & 88.10 & 87.89 \\
8 & 14089 & 22 & 2449 & 85.19 & 85.08 \\
9 & 13706 & 55 & 2474 & 84.71 & 84.42 \\
10 & 13450 & 49 & 2127 & 86.35 & 86.07 \\
\hline
\end{tabular}

Table 5. Measured Contour Area Data 4

\begin{tabular}{ccrrcc}
\hline Frame & \multicolumn{2}{c}{ Area Contour(Pixel) } & Sensitivity & Accuracy \\
Video & TP & \multicolumn{1}{c}{ FP } & \multicolumn{1}{c}{ FN } & \multicolumn{1}{c}{$(\%)$} & $(\%)$ \\
\hline 1 & 18167 & 29 & 1641 & 91.72 & 91.58 \\
2 & 17801 & 125 & 1497 & 92.24 & 91.65 \\
3 & 17258 & 24 & 2696 & 86.49 & 86.39 \\
4 & 16352 & 34 & 2038 & 88.92 & 88.75 \\
5 & 15280 & 23 & 1918 & 88.85 & 88.73 \\
6 & 14945 & 48 & 1816 & 89.17 & 88.91 \\
7 & 14093 & 95 & 1699 & 89.24 & 88.71 \\
8 & 14196 & 16 & 2077 & 87.24 & 87.15 \\
9 & 13517 & 113 & 1482 & 90.12 & 89.45 \\
10 & 13080 & 31 & 2374 & 84.64 & 84.47 \\
\hline
\end{tabular}

Table 7. Measured Contour Area Data 6

\begin{tabular}{cccccc}
\hline Frame & \multicolumn{2}{c}{ Area Contour(Pixel) } & Sensitivity & Accuracy \\
Video & TP & FP & FN & $(\%)$ & $(\%)$ \\
\hline 1 & 21469 & 220 & 1479 & 93.55 & 92.67 \\
2 & 21241 & 146 & 921 & 95.84 & 95.22 \\
3 & 20124 & 46 & 1677 & 92.31 & 92.11 \\
4 & 17726 & 9 & 2284 & 88.59 & 88.55 \\
5 & 16913 & 121 & 1717 & 90.78 & 90.20 \\
6 & 16628 & 27 & 2016 & 89.19 & 89.06 \\
7 & 15753 & 20 & 2588 & 85.89 & 85.80 \\
8 & 15479 & 4 & 2312 & 87.00 & 86.99 \\
9 & 15494 & 20 & 1600 & 90.64 & 90.53 \\
10 & 15253 & 33 & 2492 & 85.96 & 85.80 \\
\hline
\end{tabular}

\section{CONCLUSION}

This study proposes the development of a heart wall cavity tracking system automatically using optical flow. To obtain a good feature requires several stages. The first stage performs image enhancement, using a median filter with $27 \times 27$ kernels to reduce speckle noise in echocardiography images. To clarify the difference between the wall and the heart cavity, the image enhancement such as high-boost filter and morphology needs to be performed. Image segmentation stage uses global thresholding, canny, region area and collinear methods to get the contour of the heart cavity. Intersecting lines are used to define good features, which are obtained from the intersection of lines on the contour by taking a minimum line of rectangular on the left and right sides. Good features will be continued using optical flow to detect and track the movement of the heart wall. The proposed method shows the calculation results with a sensitivity of $90 \%$ and an accuracy of $87.451 \%$.

\section{ACKNOWLEDGEMENTS}

The authors would like to thank all the members of the SVG (Signal, Vision, and Graphics) laboratories and LPM Penalaran UNM for their support and guidance in this research. 


\section{REFERENCES}

[1] American Hearth Association, "What is Cardiovascular Disease," 2014.

[2] M. Seckerdieck, P. Holler, P. Smets, and G. Wess, "Simpson's method of discs in Salukis and Whippets: Echocardiographic reference intervals for end-diastolic and end-systolic left ventricular volumes," J. Vet. Cardiol., vol. 17, no. 4, pp. 271-281, 2015.

[3] M. Tawfik Ghanem, L. E. Allam, and R. Samir Ahmed, "Cardiac resynchronization therapy in patients with heart failure and moderately reduced ejection fraction: Could it trigger a super-response?," Indian Heart J., no. xxxx, pp. 4-9, 2019.

[4] N. Nakphu et al., "Apical four-chamber echocardiography segmentation using Marker-controlled Watershed segmentation," IECBES 2014, Conf. Proc. - 2014 IEEE Conf. Biomed. Eng. Sci. "Miri, Where Eng. Med. Biol. Humanit. Meet," no. December, pp. 644-647, 2014.

[5] R. Sigit, C. A. Roji, T. Harsono, and S. Kuswadi, "Improved echocardiography segmentation using active shape model and optical flow," TELKOMNIKA (Telecommunication Comput. Electron. Control., vol. 17, no. 2, pp. 809, 2019.

[6] R. Sigit, A. R. Barakbah, I. A. Sulistijono, and A. S. Aziz, "Automatic cardiac segmentation using triangle and optical flow," Indones. J. Electr. Eng. Comput. Sci., vol. 8, no. 2, pp. 315-326, 2017.

[7] R. Sigit, A. R. Barakbah, and I. A. Sulistijono, "Improved segmentation of cardiac image using triangle and partial Monte Carlo," in 2016 International Conference on Knowledge Creation and Intelligent Computing, KCIC 2016, 2017.

[8] A. Anwar, R. Sigit, A. Basuki, I. P. Adi, and S. Gunawan, "Automatic Segmentation of Heart Cavity in Echocardiography Images : Two \& Four-Chamber View Using Iterative Process Method," Proc. - Int. Electron. Symp. Knowl. Creat. Intell. Comput. IES-KCIC 2019, pp. 177-182, 2019.

[9] N. G. Pandian, D. J. Skorton, S. M. Collins, H. L. Falsetti, E. R. Burke, and R. E. Kerber, "Heterogeneity of left ventricular segmental wall thickening and excursion in 2-dimensional echocardiograms of normal human subjects," Am. J. Cardiol., vol. 51, no. 10, pp. 1667-1673, 1983.

[10] R. Sigit and E. Rochmawati, "Segmentation echocardiography video using B-Spline and optical flow," 2016 Int. Conf. Knowl. Creat. Intell. Comput. KCIC 2016, pp. 226-231, 2017.

[11] R. Sigit, T. Harsono, and B. H. A. Noor, "Heart video tracking system on long axis view," in Proceedings - 2016 International Electronics Symposium, IES 2016, 2017.

[12] A. A. Pratiwi, R. Sigit, D. K. Basuki, and Y. H. Oktaviono, "Improved ejection fraction measurement on cardiac image using optical flow," Proc. - Int. Electron. Symp. Knowl. Creat. Intell. Comput. IES-KCIC 2017, vol. 2017-January, pp. 295-300, 2017.

[13] A. S. Aziz, R. Sigit, A. Basuki, and T. Hidayat, "Cardiac motions classification on sequential PSAX echocardiogram," Indones. J. Electr. Eng. Comput. Sci., vol. 12, no. 3, pp. 1289-1296, 2018.

[14] S. S. Sengar and S. Mukhopadhyay, "A novel method for moving object detection based on block based frame differencing,” 2016 3rd Int. Conf. Recent Adv. Inf. Technol. RAIT 2016, pp. 467-472, 2016.

[15] C. M. Otto, Textbook of Clinical Echocardiography, 5th ed. Saunders, 2015.

[16] I. P. A. S. Gunawan, R. Sigit, and A. I. Gunawan, "Vein Visualization System Using Camera and Projector Based on Distance Sensor," 2018 Int. Electron. Symp. Eng. Technol. Appl. IES-ETA 2018 - Proc., pp. 150-156, 2019.

[17] S. S. Sengar and S. Mukhopadhyay, "Foreground Detection via Background Subtraction and Improved ThreeFrame Differencing," Arab. J. Sci. Eng., vol. 42, no. 8, pp. 3621-3633, 2017.

[18] Y. T. Lin and J. S. Liu, "Revisit of Minimum-Area Enclosing Rectangle of a Convex Polygon," 2018 5th Int. Conf. Control. Decis. Inf. Technol. CoDIT 2018, pp. 1051-1056, 2018.

[19] S. S. Sengar and S. Mukhopadhyay, "Moving object area detection using normalized self adaptive optical flow," Optik (Stuttg)., vol. 127, no. 16, pp. 6258-6267, 2016.

[20] R. Sigit, M. M. Mustafa, A. Hussain, O. Maskon, and I. F. M. Noh, "Automatic segmentation of cardiac cavity images using collinear and triangle equation," in Technological Developments in Networking, Education and Automation, 2010.

[21] C. Feichtenhofer and A. Pinz, "Spatio-temporal good features to track," in Proceedings of the IEEE International Conference on Computer Vision, 2013. 